\title{
Application of RFID technology in clinical monitoring system of hospital
}

\author{
Helin ZHANG ${ }^{1, a}$, Ying $Y U^{1, b}$, Shi $J^{1} N^{1, c_{*}}$ \\ ${ }^{1}$ School of computer,Jiangxi University of Traditional Chinese Medicine, Nanchang 330006, China \\ alin_he99@163.com, ${ }^{\text {b}} 59920079 @ q q . c o m,{ }^{c} 305586389 @ q q . c o m$
}

Keyword: RFID; Clinical monitoring system; Application

\begin{abstract}
In this paper, RFID is applied to the field of medical management in the field of clinical monitoring, design a set of clinical monitoring system based on the technology, to achieve the basic information on the patient read and write and query, regional positioning and access control, etc. It can improve the management efficiency of the hospital's clinical monitoring, and help to improve the prevention and health care of the disease, so as to improve the quality of hospital service.
\end{abstract}

\section{Introduction}

With the development of economy and the improvement of people's living standards, the average life expectancy of people is increasing, people's demands for medical care and hospital clinical monitoring are more and more high. According to the statistics of the Ministry of health, China's domestic more than 17000 at or above the county level medical institutions, only more than 5000 more than the municipal hospital set up his. From the real hospital comprehensive digitization and informatization construction of the overall goal, but also a difference too far. And at present, the digital information construction of the hospital is still in the primary stage, such as the clinic digital, the electronic prescription and so on.

In recent years, with the concept of the Internet of things, RFID technology has been a great development and application, the United States Intel, TI and other integrated circuit manufacturers have invested heavily in the field of RFID chip development; Phlips and other manufacturers in Europe to actively develop low-cost RFID chip, RFID recognition system, etc. Japan's main research UHF band passive and active electronic tag technology.

RFID technology has been widely used in the fields of logistics, industry and drug quality supervision. We applied the RFID technology to the design of hospital clinical monitoring system. The difficulty of RFID technology was studied. Is expected to achieve a breakthrough in the application of clinical care system in the hospital. Contribution to the construction of the digital information wisdom hospital.

\section{Application design of RFID technology in clinical monitoring system}

Presented in this paper design a based on networking RFID technology with practical hospital clinical monitoring system, design wrist wearable flexible electronic label, selected UHF frequency multi-channel read write device, the reader has better recognition communication distance, prevention of conflict of high performance, can meet the requirements of the regional hospital. Before the start of the study, through the school affiliated hospital ward field test: the use of RFID type CMC350 electronic tags, CMC165 handheld communication test, the results show that good communication effects.

The system control unit module uses embedded design, based on embedded Linux platform, SDK development kit, which is based on the embedded Linux platform, which provides the RS232 development kit, realizes the data acquisition of RFID tags, and through the PDA serial port or WLAN technology, the data is transmitted to the remote control center through wireless network.

By using the method of comparison and optimization, the anti collision algorithm and location algorithm of the electronic tags are optimized. The nearest neighbor clustering SPA relative positioning algorithm based on MATLAB is used in the LANDMARC system. The simulation 
analysis is carried out to test the performance of the algorithm.

System function module as shown below:

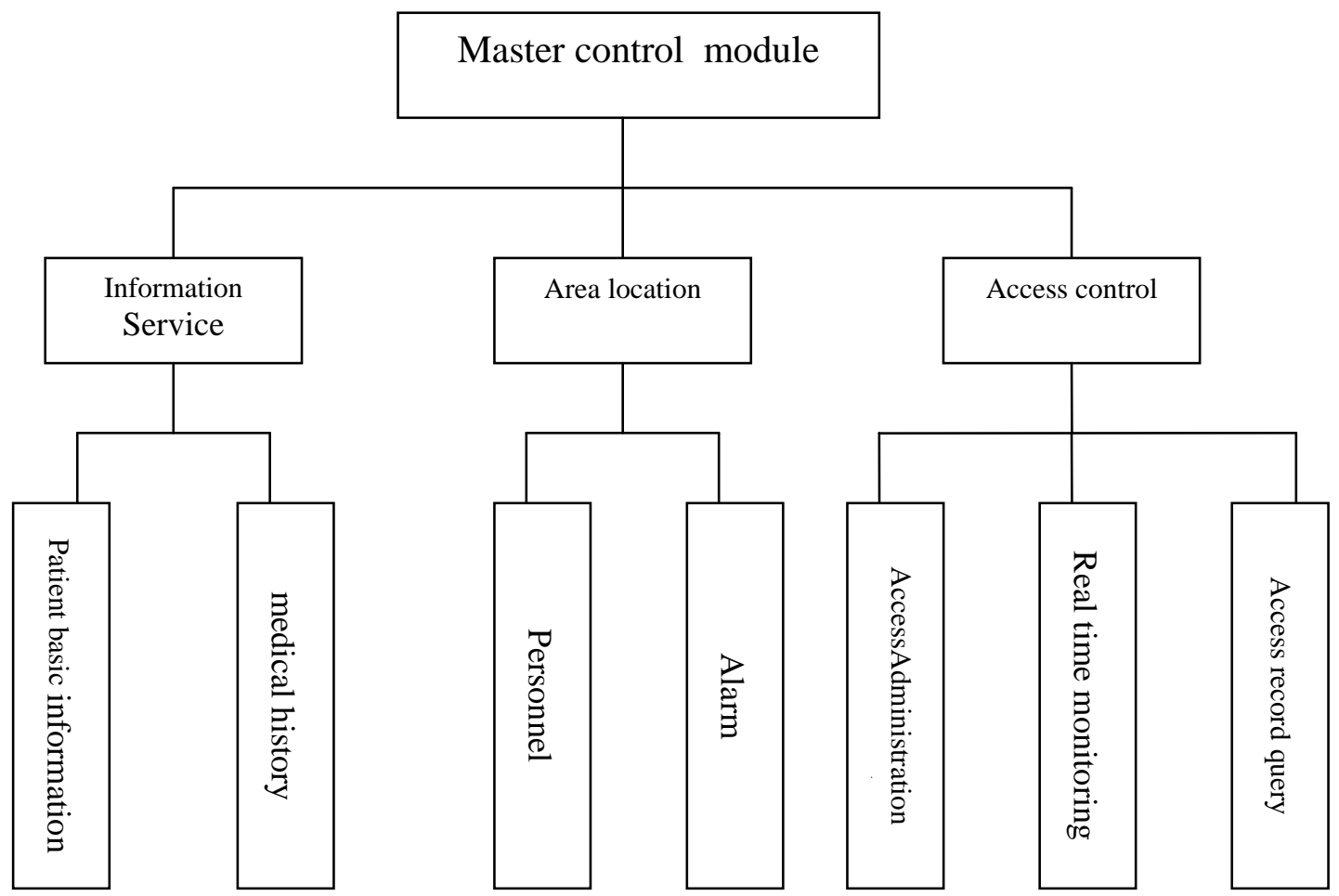

Fig.1. Functional block diagram

The main content are 3:one is Flexible design and wearable electronic tag reader in the layout design of hospital wristband can; two is Optimization research and design of anti collision algorithm and location algorithm; Three is Research on the integration of the system and the HIS system in the digital information system. Finally, the hardware and software design of the hospital clinical monitoring system based on the RFID technology of the Internet of things is complete.

\section{Hardware system design}

Specific hardware part of the main RFID transceiver unit, embedded control unit and PC unit operation unit, the structure of the block diagram is as follows:

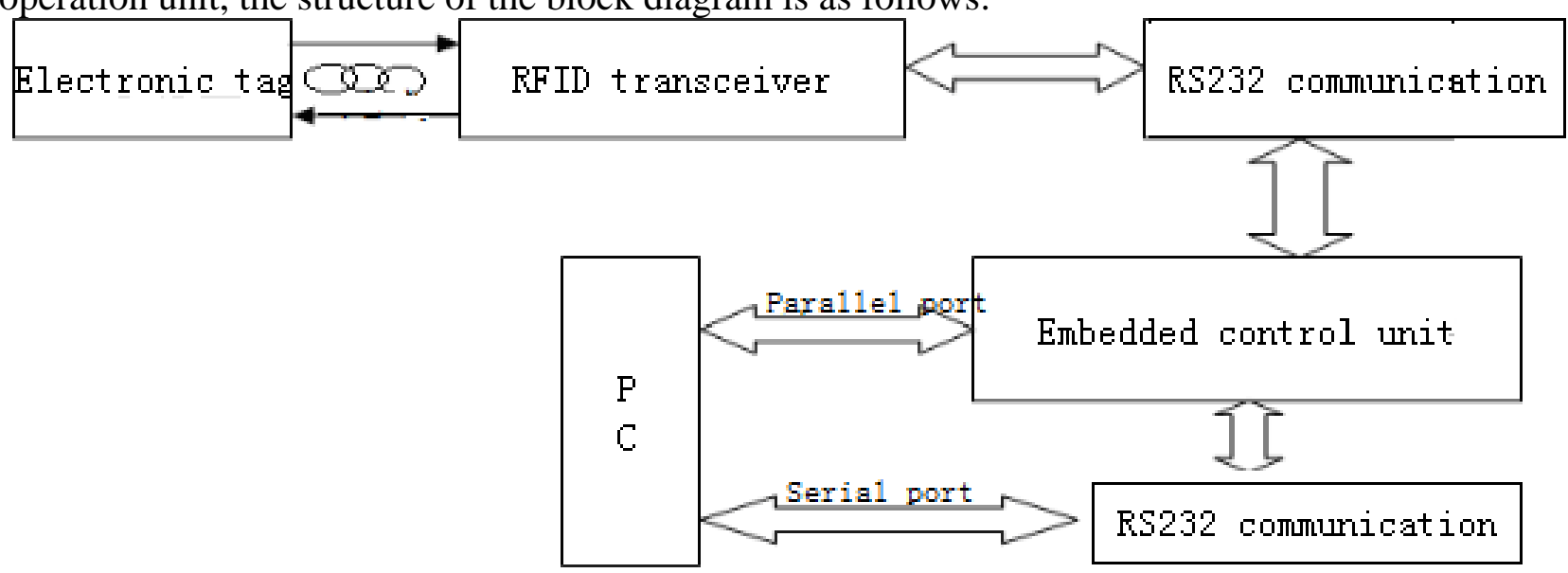

Fig.2. Hardware structure diagram

\section{Production of electronic tag}

Electronic tag production is divided into three steps: surface printing (as self-adhesive label printing), produced by the antenna and chip chip (personalized data write), self-adhesive tag printing is mainly pictures printed on the outer layer of the tag paper or plastic film, plays a 
landscaping and packaging protective effect, opened the outer, visible hidden inside the antenna (inductance) and the chip. We focus on the production of the antenna coil, select the current foreign research institutions to make the film inductance of the main winding type that is spiral coil type film inductance, which includes rectangular spiral, circular spiral, hexagonal, which is the most common form of rectangle and round, can be a good control of the individual parameters, and the shape of circular winding can greatly improve the quality factor $\mathrm{Q}$, and these two kinds of winding are relatively easy to achieve.

\section{Software design}

The clinical monitoring system based on the embedded Linux operating system platform for RFID UHF reader driver, access control system, regional positioning and RFID terminal and other clinical monitoring system software design. And to complete the development of the related graphical interface, the electronic tag data collection, transmission and storage based on RFID embedded system.

\section{Key issues in the research}

The key issues are 4: one is the system RFID technology application in multi tags under the anti collision algorithm and positioning algorithm design; two is the research and design of embedded system control module; Three is the design of flexible electronic tags and RFID handheld terminal equipment; In the end, it takes into account the feasibility of the system and the HIS system of the digital information system.

\section{Conclusions}

In this paper, a novel anti collision algorithm and location algorithm is proposed, which is based on technology to realize the control of RFID module. A hospital clinical monitoring system based on RFID technology is proposed.

\section{Acknowledgements}

This work was financially supported by the project: Health and Family Planning Commission of Jiangxi province research project (project number: 20155655)

*Corresponding author: Shi JIN, male, College of computer of Jiangxi University of Traditional Chinese Medicine , Nanchang 330006 , China; Tel: 13576976786; Email:305586389@qq.com.

\section{References}

[1] Zhou Yongbin, Feng Dengguo.RFID security protocol design and analysis of [J]. computer journal, 2006 (4).

[2] Xing Dongming, Han Jingyan, Lu Hong et al. Study on the method to evaluate the efficacy of Chinese medicine on the Journal of Harbin University of Commerce (NATURAL SCIENCE EDITION). 2001 (2): 6 - 4

[3] Chen Yan Ye Yonglin,.RFID technology application in the field of health care [J]. Technology Consulting Herald, 2007 (13).

[4] Yu Zhan Qing. Radio frequency identification (RFID) [M]. theory and application of Electronic Industry Press, 2004

[5] Hu Jia new. RFID network based on the application of hospital WLAN design [D]. Shanghai: Shanghai Jiao Tong University, 2012, 1-9 
[6] Guo Lei. Study and design of active electronic tag and antenna for RFID system [D]. Nanjing: Nanjing University of Aeronautics \&amp; Astronautics, 2012, 4 - 